\title{
Monte Carlo Simulations in Solar Radio Astronomy
}

\author{
G. Thejappa ${ }^{1}$ and R. J. MacDowall ${ }^{2}$ \\ ${ }^{1}$ Department of Astronomy University of Maryland College Park MD 20742 \\ ${ }^{2}$ NASA/Goddard Space Flight Center Greenbelt MD 20771
}

USA

\section{Introduction}

Propagation effects, especially, the refraction by the slowly varying ambient plasma, and the scattering by the random density fluctuations are known to distort the characteristics of the low frequency solar radio emissions. At kilometric radio wavelengths, they are probably responsible for the higher apparent source heights, larger source sizes, and widespread visibilities of type III radio bursts, and anomalous propagation time delays between signals arriving at widely separated spacecraft (Lecacheux et al , 1989; Steinberg et al , 1984; 1985; Thejappa et al , 2007). The scattering is also probably responsible for the low brightness temperatures and large equatorial diameters of the quiet sun (Aubier et al , 1971; Thejappa \& Kundu , 1992; 1994; Thejappa and MacDowall , 2008a;b).

The regular refraction of radio waves in a spherically symmetric solar atmosphere has been investigated using the ray tracing methods (Bracewell \& Preston, 1956; Jaeger \& Westfold, 1950; Smerd , 1950; Thejappa and MacDowall , 2010). When the influence of plasma on wave propagation can be neglected, for example, in the case of interplanetary scintillations, the scattering and diffraction can be studied using parabolic equation methods (Bastian , 1994; Cairns , 1998; Lee \& Jokipii, 1975; Rickett , 1977; Rytov et al , 1989). When the plasma can have a significat effect on the propagation, the geometric optics method is usually used to study the scattering of the solar radio emissions (Cairns ,1998; Thejappa et al ,2007). The scattering by multiple, independent and, random distribution of inhomogeneities is usually simulated using the statistical ray tracing techniques (Fokker , 1965; Hollweg , 1968; Riddle , 1974b; Steinberg et al , 1971; Steinberg , 1972). The geometric optics method treats the scattering as irregular refractions of rays, and introduces it as a random perturbation to the direction of the wave propagation vector. Such treatment is valid as long as the rms phase fluctuation $\delta \phi=\frac{2 \pi}{\lambda} \delta \mu \Delta S$ is not greater than a radian and the mean refractive index $\mu$ is constant, so that the ray remains quasi-linear. Here, the $\lambda$ is the wavelength, $\mu$ is the refractive index, and $\Delta S$ is the path length. The Monte Carlo methods are used to compute these small random perturbations in the directions of the rays due to scattering. For each scattering event, so that the scattering angles $\left\langle\psi^{2}\right\rangle=2 \sqrt{\pi} \int_{\text {ray }} \frac{\left\langle\delta \mu^{2}\right\rangle}{\mu^{2} l} d S$ are small and the rays remain quasilinear, the ray path is divided into linear steps of $\Delta S$ chosen in such a way that the conditions $\psi<0.1$ radians, and $\frac{\Delta \mu}{\mu}<0.1$ are satisfied over each step. Here $l$ is the scale length of the density fluctuations. 
Fokker (1965) used the Monte Carlo technique to compute the sizes of the scatter images, intensity distributions, and directivities of type I solar radio bursts. By including the regular refraction into these statistical raytracing techniques, Steinberg et al (1971) and Riddle (1974b) studied the effects of scattering on the metric type III radio bursts. Steinberg (1972) used this technuque to compute the directivity of type III bursts at $3 \mathrm{MHz}$, and compared with the observed center-limb histograms obtained by Fainberg \& Stone (1970). The Monte Carlo methods are also used to study several other problems in solar radio astronomy (Aubier et al , 1971; Caroubalos et al , 1972; Hoang \& Steinberg , 1977; Leblanc , 1973; Riddle , 1974a; Thejappa \& Kundu , 1992; 1994; Thejappa et al , 2007; Thejappa and MacDowall , 2008a;b). For example, Aubier et al (1971) have shown that the scattering is probably responsible not only for lowering the brightness temperatures of the quiet sun radio emission at metric and decametric wavelengths, but also for raising their apparent source sizes. Thejappa \& Kundu (1992) have shown that the scattering can lower the brightness temperatures of the quiet sun component to the observed values of $\sim 10^{5} \mathrm{~K}$, if the relative level of density fluctuations, $\epsilon=\frac{\Delta N_{e}}{N_{e}}$ is at least of the order of $\simeq 0.1$. The main criticism of these studies is that they assume (1) idealized spherically symmetric density models for the solar atmosphere, and a Gaussian spectrum for the electron density fluctuations, and (2) arbitrary values for the relative level of density fluctuations, $\epsilon$, and their spatial scales, $l$. Several decades of in situ turbulence and interplanetary scintillation studies have yielded that the spatial power spectrum of density fluctuations is of a power-law type (Coles \& Harmon, 1989; Coles et al , 1991). Similar extensive eclipse and coronograph observations have shown that the spherically symmetric models for the electron density are highly idealized.

We have developed an efficient Monte-Carlo simulation technique, and applied it to study the directivity, visibility, time profiles, source sizes, and East-West asymmetries of low frequency type II and type III radio bursts (Thejappa et al , 2007; Thejappa and MacDowall , 2008b). We have also investigated the effects of refraction and scattering on the quiet sun radio emission (Thejappa and MacDowall , 2008a;b). Since, in the lower solar corona, the power is concentrated mainly in the flat part of the power spectrum with spectral index, $\alpha=3$, we have derived an expression for the angular deflection suffered by a ray due to scattering by such density fluctuations in a slab of thickness, $\Delta S$. Using realistic models for the electron density, and density fluctuations, and observed values for $\epsilon$, and $l$, we statistically derive the emission characteristics of radio bursts, and quiet sun, and compare them with observations.

\section{Model}

\subsection{Electron density}

For the solar wind, we use the empirical formula derived by Bougeret et al (1984b)

$$
N_{e}(r)=6.14 r^{-2.10} \mathrm{~cm}^{-3},
$$

where $r$ is the heliocentric distance in units of AU. For the quiet sun, we use the empirical formula derived by Guhathakurta et al (1996), based on Skylab data obtained during the declining phase of solar cycle 20 (1973-1976)

$$
N_{e}\left(r, \theta_{m g}\right)=N_{p}(r)+\left[N_{c s}(r)-N_{p}(r)\right] e^{-\theta_{m g}^{2} / w^{2}(r)} \mathrm{cm}^{-3},
$$

where $r$ is the heliocentric distance in units of $R_{\odot}, N_{c S}(r)$ and $N_{p}(r)$ are the electron densities at the current sheet and the poles, respectively, $w(r)$ is the half-angular width of the current 
sheet, $\theta_{m g}$ is the angular distance of a point from the current sheet in the heliomagnetic coordinate system (heliomagnetic latitude). The $\theta_{m g}$ is given by

$$
\theta_{m g}=\sin ^{-1}\left[-\cos \theta \sin \alpha \sin \left(\phi-\phi_{0}\right)+\sin \theta \cos \alpha\right],
$$

where $\theta$ and $\phi$ are the heliographic latitude and longitude, respectively, $\alpha \simeq 15^{\circ}$ is the tilt angle of the dipole axis with respect to the rotation axis, and $\phi_{0} \simeq 0$ is the angle between the heliomagnetic and heliographic equators. The $N_{\mathcal{C S}}(r)$ and $N_{p}(r)$ are defined as

$$
N_{e}(r)=\Sigma_{i=1}^{3} c_{i} r^{-d_{i}}
$$

where $c_{1}, c_{2}$ and $c_{3}$ are 1.07, 19.94, and 22.10 for the current sheet, and $0.14,8.02$, and 8.12 for the pole, respectively. These coefficients are in units of $10^{7}$. The coefficients $d_{1}, d_{2}$ and $d_{3}$ are $2.8,8.45$, and 16.87, respectively. The functional form of $w(r)$ is

$$
w(r)=\Sigma \gamma_{i} r^{-\delta_{i}}
$$

where $\gamma_{1}=16.3^{\circ}, \gamma_{2}=10^{\circ}, \gamma_{3}=43.2^{\circ}, \delta_{1}=0.5, \delta_{2}=7.31$, and $\delta_{3}=7.52$. We neglect the ambient magnetic field, because the electron cyclotron frequency $f_{c e}$ is usually much less than the electron plasma frequency $f_{p e}$. We assume that the electron temperature $T_{e}$ is $1 \times 10^{6} \mathrm{~K}$ in the solar corona and $1.5 \times 10^{5} \mathrm{~K}$ in the solar wind. We define the $f_{p e}$, the refractive index $\mu$, and the electron collision frequency $v$ as

$$
\begin{array}{r}
f_{p e}^{2}=80.6 \times 10^{6} N_{e}, \\
\mu^{2}=1-\frac{f_{p e}^{2}}{f^{2}}, \\
v=4.36 N_{e} T_{e}^{-3 / 2}\left[17.72+\ln \left(T_{e}^{3 / 2} / f\right)\right],
\end{array}
$$

where $f$ is the frequency in $\mathrm{Hz}$.

\subsection{Electron density fluctuations}

The observations show that the spatial power spectrum of density fluctuations is of the power law type (Coles \& Harmon, 1989; Coles et al , 1991)

$$
P_{n}(q)=C_{N}^{2} q^{-\alpha} ; q_{o}<q<q_{i}
$$

where $q$ is the spatial wavenumber, $\alpha$ is the spectral index, and $l_{0}=2 \pi q_{o}$ and $l_{i}=2 \pi q_{i}$ are the outer and inner scales of the density turbulence, respectively. Coles \& Harmon (1989) have shown that for scales larger than a few times $100 \mathrm{~km}, \alpha$ is 11/3 (Kolmogorov spectrum), for intermediate scales (a few $\mathrm{km} \leq l \leq$ few times $100 \mathrm{~km}$ ) $\alpha$ changes from 11/3 to $\sim 3$ (flat spectrum), and for the smallest scales of $\sim 2 \mathrm{~km}$ (inner or dissipative scales) the spectrum becomes quite steep with $\alpha \simeq 4$. The power is mainly concentrated in the flat part of the spectrum (Coles et al, 1991). By normalizing the spectrum to the variance of density fluctuations $\left\langle\Delta N_{e}^{2}\right\rangle$, the expression for the structural constant $C_{N}^{2}$ can be written as (Efimov et al, 2005)

where

$$
C_{N}^{2}=A\left(\alpha, q_{0}, q_{i}\right)<\Delta N_{e}^{2}>
$$

$$
A\left(\alpha, q_{0}, q_{i}\right)= \begin{cases}\frac{(\alpha-3) \Gamma(\alpha / 2) q_{o}^{\alpha-3}(2 \pi)^{-3 / 2}}{\Gamma[(\alpha-1) / 2]} & \text { for } 3<\alpha<4, \\ \frac{1}{4 \pi \ln \left(\frac{2 q_{0}}{q_{i}}\right)} & \text { for } \alpha=3 .\end{cases}
$$


For a Kolmogorov spectrum with $\alpha=11 / 3$, this expression takes the form

$$
C_{N}^{2}=\frac{\epsilon^{2} l_{o}^{-2 / 3} N_{e}^{2}}{6.6}
$$

where $\epsilon=\frac{\Delta N_{e}}{N_{e}}$. This expression agrees approximately with $C_{N}^{2}=\frac{\epsilon^{2} l_{0}^{-2 / 3} N_{e}^{2}}{5.53}$, derived by Spangler (2002). For the flat spectrum with $\alpha=3$, this becomes

$$
C_{N}^{2}=\frac{\epsilon^{2} N_{e}^{2}}{4 \pi \ln \left(\frac{2 l_{i}}{l_{o}}\right)} .
$$

For the solar wind, $\alpha \sim 11 / 3$ agrees with that of Kolmogorov derived for fluid turbulence (Spangler \& Sakurai, 1995; Spangler et al , 2002; Spangler , 2002; Tu \& Marsch , 1994; Wohlmuth et al , 2001; Woo et al , 1995). The inner scale $l_{i}$ (which is also known as the dissipative scale) increases linearly with heliocentric distance as $l_{i}=\left(\frac{R}{R_{\odot}}\right)^{ \pm 0.1} \mathrm{~km}$ at $R \leq 100 R \odot$ and from 100 to $200 \mathrm{~km}, l_{i} \simeq 90-100 \mathrm{~km}$ (Coles \& Harmon, 1989; Manoharan et al , 1988). In this study, we assume that $l_{i} \sim 100 \mathrm{~km}$, and for the outer scale $l_{0}$, we use the empirical formula derived by Wohlmuth et al (2001) using the Galileo data between 7 to $80 R \odot$

$$
l_{0}=19 r^{0.82} \text {. }
$$

Here we have rewritten the empirical relation of Wohlmuth et al (2001) in units of AU. Based on Helios observations, Bavassano \& Bruno (1995) deduced that most of the time $\epsilon$ is 0.07 and is 0.1 for $14 \%$ of the time. In this study, we assume that $\epsilon$ is 0.07 through out the solar wind (Cairns, 1998).

For the quiet sun studies, we consider the spatial scales, which range from 50 to $75 \mathrm{~km}$. For $l_{i}=50 \mathrm{~km}, l_{o}=75 \mathrm{~km}$ and $\alpha=3$, we obtain from (12)

$$
C_{N}^{2}=0.28 \epsilon^{2} N_{e}^{2} \text {. }
$$

The radio scattering observations indicate that the coronal and solar wind turbulence is highly anisotropic (see, for example, Armstrong et al (1990); Coles et al (2002); Grall et al (1997); Narayan et al (1989)). For example, Coles et al (2002) have given an empirical formula for the axial ratio, AR as

$$
(A R-1) \simeq \frac{160}{r^{3 / 2}}
$$

where $\mathrm{r}$ is in units of solar radii $R \odot$. At heliocentric distances corresponding to meter and decameter wavelength radio emissions, we assume that the spatial scales along the magnetic field are 10 times larger than those perpendicular to the field. We also assume that $\epsilon=0.1$ throughout the corona.

\section{Monte Carlo method}

\subsection{Ray tracing}

In the Cartesian coordinate system with origin at the center of the Sun, and the x-axis coinciding with the radial direction, the following set of 6 first-order differential equations describe the ray tracing (Haselgrove, 1963)

$$
\begin{array}{r}
\frac{d \vec{R}}{d \tau}=\vec{T} \\
\frac{d \vec{T}}{d \tau}=D(\vec{R})=\frac{1}{2} \frac{\partial \mu^{2}}{\partial \vec{R}},
\end{array}
$$


with

$$
T_{x}^{2}+T_{y}^{2}+T_{z}^{2}=\mu
$$

Here

$$
\vec{R} \equiv\left(\begin{array}{c}
x \\
y \\
z
\end{array}\right) \text { and } \vec{T} \equiv\left(\begin{array}{c}
T_{x} \\
T_{y} \\
T_{z}
\end{array}\right)
$$

are the position and direction vectors of the ray, respectively. The independent variable $\tau$ is related to actual path length $s$ as

$$
d \tau=\frac{d s}{\mu} .
$$

Using equations (6) and (7), we can write

$$
D(\vec{R}) \equiv \frac{1}{2}\left(\begin{array}{l}
\frac{\partial \mu^{2}}{\partial x} \\
\frac{\partial \mu^{2}}{\partial y} \\
\frac{\partial \mu^{2}}{\partial z}
\end{array}\right)
$$

as

$$
D(\vec{R})=\frac{8.90 \times 10^{12}}{f^{2}} \frac{1}{r^{4}} N_{e} \vec{R} .
$$

We use the Runge-Kutta algorithm to integrate the ray tracing equations (16) and (17), which can be written in vectorial form as (see for example Sharma et al (1982))

$$
\begin{array}{r}
R_{n+1}=R_{n}+\Delta \tau\left[T_{n}+\frac{1}{6}(A+2 B)\right], \\
T_{n+1}=T_{n}+\frac{1}{6}(A+4 B+C), \\
A=\Delta \tau D\left(R_{n}\right), \\
B=\Delta \tau D\left(R_{n}+\frac{\Delta \tau}{2} T_{n}+\frac{1}{8} \Delta \tau A\right), \\
C=\Delta \tau D\left(R_{n}+\Delta \tau T_{n}+\frac{1}{2} \Delta \tau B\right), \\
D(R)=\frac{1}{2} \frac{\partial \mu^{2}}{\partial R} .
\end{array}
$$

We compute the optical depth $\tau$ and the transit time $\Delta t(s)$ at each step $\Delta S$ on the ray path as

$$
\begin{gathered}
\tau_{i+1}=\tau_{i}+K \frac{f_{p e}^{2}}{f^{2}} \frac{v \Delta S}{\mu_{i}} \\
\Delta t_{i+1}=\Delta t_{i}+K \frac{\Delta S}{\mu_{i}},
\end{gathered}
$$

where $\mathrm{K}$ is 500 , (1 A. U devided by velocity of light) in the solar wind, and 2.32 (solar radius divided by velocity of light) in the solar corona. Using this algorithm, one can trace the rays through any medium, i.e., starting from a known point $\left(\vec{R}_{0}, \vec{T}_{0}\right)$, one can generate successively $\left(\overrightarrow{R_{1}}, \vec{T}_{1}\right),\left(\overrightarrow{R_{2}}, \vec{T}_{2}\right) \ldots . .\left(\vec{R}_{n}, \vec{T}_{n}\right)$. 


\subsection{Scattering}

The components of $\vec{T}$ (eqn. 22) are the direction cosines of the ray after it has suffered a regular refraction in a layer of thickness $\Delta S$. The scattering by random density fluctuations in this layer, i.e., the random perturbation vector $\langle\vec{p}\rangle$ is added to $\vec{T}$ at each step. The components of $\left\langle\vec{p}>\right.$ are computed using the mean-square angular deviation $\left\langle\Psi^{2}>\right.$ suffered by the ray due to scattering in the layer of $\Delta S$. Chadrasekhar (1952) and Hollweg (1968) have derived the expression

$$
<\Psi^{2}>=b(f) \Delta S
$$

where $b(f)$ is the mean square deviation per unit length. For the power spectrum $P_{n}(q)=$ $C_{N}^{2} q^{-\alpha}$, the expression for the mean-square angular deviation is (Cairns , 1998; Thejappa et al ,2007)

$$
<\Psi^{2}>=\frac{r_{e}^{2} \lambda^{4}}{\pi \mu^{2}} \Delta S \frac{C_{N}^{2}}{4-\alpha}\left(q_{i}^{4-\alpha}-q_{o}^{4-\alpha}\right) .
$$

For $q_{0}<<q_{i}$, this expression can be simplified as

$$
<\Psi^{2}>=\frac{r_{e}^{2} \lambda^{4}}{\pi \mu^{2}} \Delta S C_{N}^{2} \frac{q_{i}^{4-\alpha}}{4-\alpha} .
$$

For $\alpha=11 / 3$ (spectral index), $r_{e}=\frac{e^{2}}{m c^{2}}$ (the classical radius of the electron), $\lambda=\frac{c}{f}$ (the wavelength of the wave in free space), $C_{N}^{2}=\frac{\epsilon^{2} l_{0}^{-2 / 3} N_{e}^{2}}{5.53}$, (structural coefficient), and $f_{p e}^{2}=\frac{e^{2} N_{e}}{\pi m_{e}}$ (the electron plasma frequency), we obtain from equations (29) and (31)

$$
b(f)=\pi \frac{f_{p e}^{4}}{f^{4}} \frac{\epsilon^{2}}{\mu^{4} l_{i}^{1 / 3} l_{o}^{2 / 3}} .
$$

For the flat spectrum with $\alpha=3$, and for $Q_{i}=\frac{2 \pi}{L_{i}}$ and $Q_{o}=\frac{2 \pi}{L_{o}}$ (corresponding to the lower and upper limits of the range of the considered spatial scales in the place of $q_{i}$ and $q_{o}$ ) we obtain from equation (30)

$$
b(f)=\pi \frac{\left(1-L_{i} / L_{o}\right)}{2 \ln \left(2 L_{i} / L_{o}\right)} \frac{f_{p e}^{4}}{f^{4}} \frac{\epsilon^{2}}{\mu^{4} L_{i}},
$$

where, we have used the expression $C_{N}^{2}=\frac{\epsilon^{2} N_{e}^{2}}{4 \pi \ln \left(2 L_{i} / L_{o}\right)}$. For $L_{i}=50 \mathrm{~km}$, and $L_{0}=75 \mathrm{~km}$, we obtain from equation (33)

$$
b(f) \sim 0.6 \pi \frac{f_{p e}^{4}}{f^{4}} \frac{\epsilon^{2}}{\mu^{4} L_{i}} .
$$

For Gaussian fluctuations, the expression for $b(f)$ (Lacombe et al , 1988)

$$
b(f)=\frac{\sqrt{\pi}}{\mu^{4}} \frac{f_{p e}^{4}}{f^{4}} \frac{\epsilon^{2}}{h},
$$

coincides with the expression (32) derived for the Kolmogorov spectra for an effective scale height $h=l_{i}^{1 / 3} l_{0}^{2 / 3}$. Similarly, the expression (34) derived for $\alpha=3$ coincides with (35) derived for the Gaussian spectrum. The components of $\langle\vec{p}\rangle$ are chosen from a Gaussian distribution of random numbers with a zero mean and a standard deviation of

$$
\sigma=\mu \sqrt{b \Delta S}
$$


For isotropic fluctuations, three independent Gaussian distributed random deviations of the direction cosines with the same standard deviation (36) are calculated. However, for anisotropic fluctuations with longitudinal scales much larger than the transverse scales, $\sigma$ changes accordingly, with $\sigma_{\|}<\sigma_{\perp}$, since $\sigma$ is inversely proportional to the square root of the spatial scale of the density fluctuations.

\section{Type III radio bursts}

By assuming that both fundamental and harmonic emissions are emitted by isotropic point sources, we can assign the initial directions of the rays so that they end on a sphere of radius equal to the local $\mu_{0}$. Then, the probability $p\left(\theta_{0}, \phi_{0}\right)$, that a point belongs to an element of a spherical surface $\sin \theta_{0} d \theta_{0} d \phi_{0}$ is $\frac{\sin \theta_{0} d \theta_{0} d \phi_{0}}{4 \pi}$, where $0 \leq \theta_{0} \leq \pi$ and $0 \leq \phi_{0} \leq 2 \pi$. By writing $p\left(\theta_{0}, \phi_{0}\right)=p_{1}\left(\theta_{0}\right) p_{2}\left(\phi_{0}\right)$, we obtain $p_{1}\left(\theta_{0}\right)=\frac{\sin \theta_{0}}{2}$ and $p_{2}\left(\phi_{0}\right)=\frac{1}{2 \pi}$, and from the integrals $\int_{0}^{\phi_{0}} p_{2}\left(\phi_{0}\right) d \phi_{0}=\frac{\phi_{0}}{2 \pi}=\xi_{1}$, and $\int_{0}^{\theta_{0}} p_{1}\left(\theta_{0}\right) d \theta_{0}=\frac{1}{2} \int_{0}^{\theta_{0}} \sin \theta_{0} d \theta_{0}=\frac{\cos \theta_{0}}{2}+\frac{1}{2}=\xi_{2}$, we obtain the azimuthal and elevation angles of the initial ray directions as

$$
\begin{array}{r}
\phi_{0}=2 \pi \xi_{1} \\
\cos \theta_{0}=2 \xi_{2}-1,
\end{array}
$$

where $\xi_{1}$ and $\xi_{2}$ are the random variables distributed uniformly between 0 and 1 . Thus, the optical direction cosines of initial rays can be written as

$$
\begin{array}{r}
T_{x 0}=\mu_{0} \sin \theta_{0} \sin \phi_{0} \\
T_{y 0}=\mu_{0} \sin \theta_{0} \cos \phi_{0} \\
T_{z 0}=\mu_{0} \cos \theta_{0},
\end{array}
$$

which imply that $-\mu_{0} \leq\left(T_{x 0}, T_{y 0}, T_{z 0}\right) \leq \mu_{0}$. By assigning these random directions for the directions of initial rays, we eliminate the possibility for any two rays to have the same initial direction.

We have developed a computer program based on the algorithm presented in the previous section. We have used it to trace the rays statistically by assuming that both fundamental and harmonic emissions are emitted at $120 \mathrm{kHz}$, corresponding to altitudes of $0.2097 \mathrm{AU}$ $\left(f_{p e} \sim 115\right)$ and $0.3895 \mathrm{AU}\left(f_{p e}=60 \mathrm{kHz}\right)$, respectively. We have considered two cases (1) $\epsilon=0$ and (2) $\epsilon=0.07$. For each case, we launch 1000 randomly directed rays and trace them until they cross the sphere of $1 \mathrm{AU}$ radius. For $\epsilon=0$, when only the regular refraction is considered, we take $\Delta S=0.002 \mathrm{AU}$, and for $\epsilon=0.07$, when both regular refraction and scattering are dominant we take $\Delta S=10 l$, where $l=l_{i}^{1 / 3} l_{0}^{2 / 3}$. At the exit point, we record the components of $(\vec{R})$ and $(\vec{T})$, total optical depth $\tau$ and time delay $\Delta t$ (calculated using equations 27 and 28) in a separate file. These recorded values will be used to calculate the directivities, time profiles, and sizes and heights of the apparent sources of both fundamental and harmonic emissions. In Fig. 1, we present the typical trajectories of the traced rays. Here, the first and second columns correspond to the fundamental and harmonic emissions, whereas the first and second rows correspond to the cases, $\epsilon=0$, and $\epsilon=0.07$, respectively. We have embedded the distributions of the ray trajectories inside the transparent spheres of $1 \mathrm{AU}$ radius to have a better representation of the Sun, the rays emanating from the source, and the observer. The distributions of the rays in the first row show that the regular refraction focuses the fundamental into a narrower cone than that of the harmonic, and the distributions in the second row show that the scattering $(\epsilon=0.07)$ destroys the refractive focusing. 

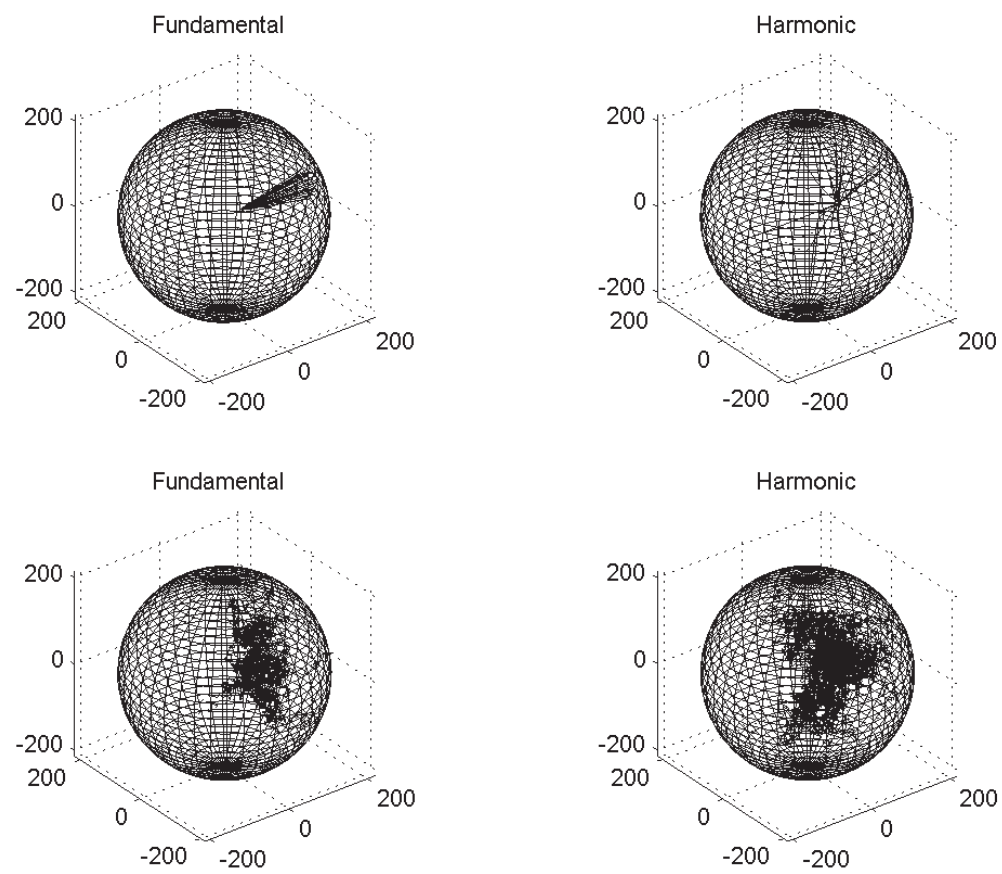

Fig. 1. Typical distributions of the traced rays of the fundamental (first column) and harmonic (second column) emissions. The first row corresponds to the case of only regular refraction, whereas the second row corresponds to the regular refraction as well as scattering.

\subsection{Directivity}

Each ray is characterized by an angle $\beta$ subtended at the center by the traced portion of the ray from the source to $1 \mathrm{AU}$. Since in the spherically symmetric case, the axis through the source and the center of the Sun is also the axis of the cylindrical symmetry, the angle $\beta$ determines the distance of the apparent source from the center of the solar disk. In heliographic degrees, $\beta$ is defined as

$$
\beta=\frac{\cos ^{-1}(\vec{R} \cdot \vec{x})}{|\vec{R}|},
$$

where the $\mathrm{x}$-axis is the axis of symmetry, and $\vec{R}$ is the position vector at the exit point. For a source on the solar equator, $\beta$ is the longitude measured from the central meridian. The directivity is defined as the ratio of the power received (i.e., number of rays) in a range of angles from $\beta$ to $\beta+d \beta$ from the source embedded in a scattering and refracting medium, to the power received from the same source at the same position emitting the same total power isotropically in a vacuum. This can be expressed as a ratio of the total number of rays in a group of angles centered around $\beta$ each weighted by $e^{-\tau}$, to the total number of rays that would fall in the same group of $\beta$ from an isotropic source when the ray paths are unaffected by either scattering or refraction, i.e.,

$$
D(\beta)=\frac{4 \pi \Sigma_{n_{\beta}} e^{-\tau_{i \beta}}}{\Delta \Omega N_{T}},
$$


where $n_{\beta}$ is the number of rays escaping in the angles from $\beta$ to $\beta+d \beta$, and $N_{T}$ is the total number of rays. The total optical depth $\tau_{\beta}$ along each ray is computed by summing the optical depths along all the steps taken by the ray from the source to its exit as given by equation (27). The attenuation coefficient $e^{-\tau}$ represents the losses suffered by the ray due to increased path lengths caused by scattering. The solid angle $\Delta \Omega$ spanned by grid separation in the $\beta$ direction around the annular ring is defined as

$$
\Delta \Omega=4 \pi \sin \left[\left(i_{\beta}+0.5\right) \Delta \beta\right] \sin (\Delta \beta / 2),
$$

where $\Delta \beta$ and $i_{\beta}$ are the angular width and index of the group, respectively. We have computed the directivities for $\epsilon=0$ as well as for $\epsilon=0.07$ by counting the number of harmonic and fundamental rays in groups of 5 and 1 degree intervals, respectively, and normalized them by dividing each of them by the largest in each case. In Fig. 2, we present these normalized directivities, where the first row clearly shows that in a smoothly varying plasma $(\epsilon=0)$, the refraction focuses the fundamental and harmonic emissions into cones of $\sim 18^{\circ}$ and $\sim 80^{\circ}$ angular widths, respectively. The refractive focusing can be understood in
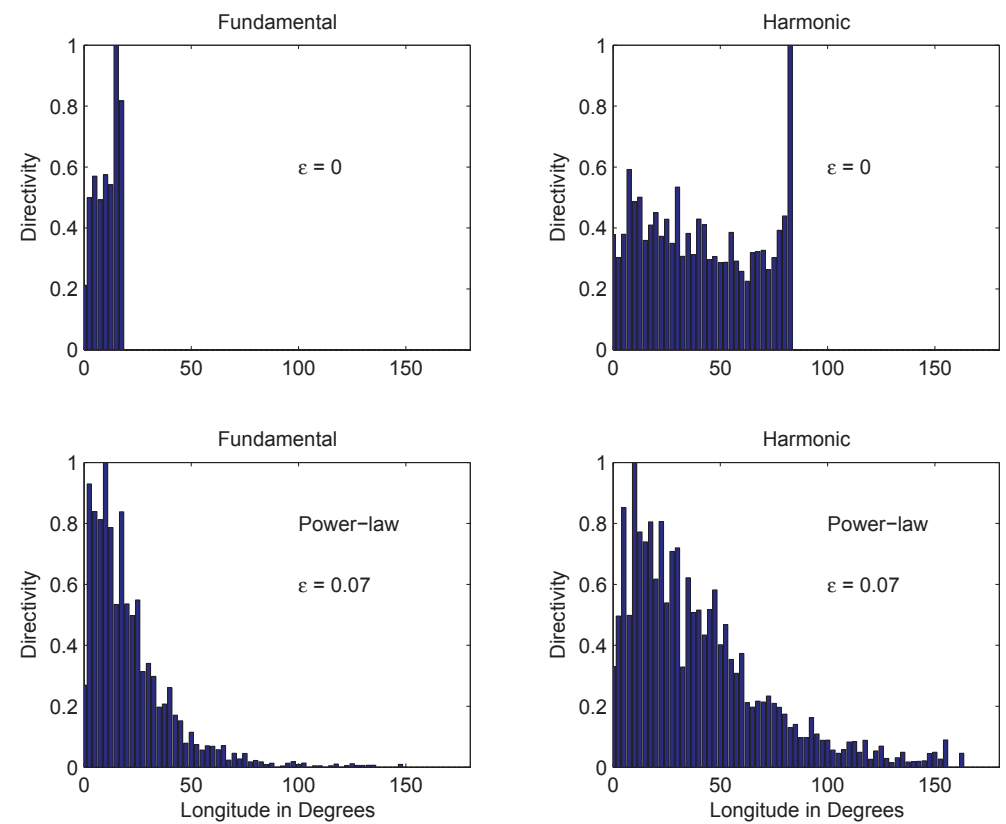

Fig. 2. The left and right columns show the directivities of the fundamental and harmonic emissions, respectively. Here $\epsilon$ is equal to the level of relative density fluctuations $\frac{\Delta N_{e}}{N_{e}}$ terms of the law of refraction in a plane-layered plasma

$$
\mu(z) \sin \phi=\mu\left(z_{0}\right) \sin \phi_{0},
$$

where $\phi$ is the angle between the ray and the gradient of the refractive index. At the exit point $z=z_{0}$, the refractive index $\mu\left(z_{0}\right)$ is $\sim 1$ and the angle $\phi$ is $\phi_{0}$, and at the point of reflection $z=z_{\text {ref }}$ the angle $\phi$ is $\pi / 2$ and (from equation (45))

$$
\mu\left(z_{r e f}\right)=\sin \phi_{0} .
$$


For an isotropic point source, the refraction bends the rays into a cone of angular width $\phi_{0}$. The apex of the ray leaving the plasma at the maximum angle $\phi_{0}=\phi_{\max }$ coincides with the source location $z=z_{s}$. The angular width of the limiting cone can be obtained from equation (46) as

$$
\phi_{\text {max }}=\sin ^{-1} \mu\left(z_{s}\right)=\sec ^{-1}\left(f / f_{p e}\right) .
$$

For example, $\phi_{\max }$ is $5^{\circ}, 16.6^{\circ}$, and $60^{\circ}$, for $f \sim 1.004 f_{p e}, f \sim 1.0435 f_{p e}$, and $f=2 f_{p e}$, respectively. The computed limiting cones (see first row of Fig. 2) are slightly broader than those estimated using equation (47), because in the computations spherical symmetric model is used, whereas the $\phi_{\max }$ in equation (47) is derived using the plane parallel approximation. The intense "shoulders" at the edges of the limiting cones seen in the directivity diagrams (first row of Fig. 2) at $\simeq 18^{\circ}$ and $\simeq 80^{\circ}$ longitudes probably are due to ingoing rays from the source (Steinberg et al , 1971; Steinberg, 1972).

We use the histograms presented in row 1 to compute the directivity factors as the ratios of intensities at two different longitudes. For $\epsilon=0$, the ratio of intensities at $5^{\circ}$ and $15^{\circ}$ is 0.5 for the fundamental, and the ratio of intensities at $5^{\circ}$ and $80^{\circ}$ longitudes is 0.75 for the harmonic. For $\epsilon=0$, the fundamental emission is very intense and directive for an observer located within its limiting cone in comparison with that of the harmonic, by indicating that during low level of density fluctuations, the positive identification of the mode of emission of the observed type III or the type II bursts with the fundamental, or a mixture of strong fundamental and weak harmonic emissions is a good indication of an imminent arrival of the flare accelerated electrons or the CME driven shock accelerated electrons at the spacecraft.

\subsection{Time profiles}

The arrival times of scattered rays ultimately received in a given direction are different for different rays. If the source radiates a very short pulse, the intensity recorded as a function of time is the "transient" response of the ambient medium. Thus, the observed time profile can be understood as the convolution of the time profile at the source and the time response at $1 \mathrm{AU}$ of an impulsive burst at the source. The ambient medium refracts and scatters the ray from the time it is launched until it exits the medium. The time taken by the ray during each step $\Delta t_{i}$ is $\frac{S_{i}}{c \mu_{i}}$, where $c$ is the velocity of light, $S_{i}$ is the path length traveled by the ray during the $i$-th step, and $\mu_{i}$ is the refractive index; the $c \mu_{i}$ is the group speed of the ray. The time taken by the ray to travel from the source to the point of exist from the medium (arrival time) is the sum of all the time steps $\Delta t_{i}$ as given in equation (28). The histogram of these arrival times at $1 \mathrm{AU}$ gives the time profile of the isotropic point source. In the first column of Fig. 3, we present the time profiles of the fundamental constructed using the arrival times of the rays gathered in the longitude range from 0 to $30^{\circ}$. These show that the total durations of the unscattered and scattered emissions are 50 (top panel), and $\sim 3000$ seconds (bottom panel), respectively. The time profile of the scattered emission, which is characterized by a rapid rise followed by an exponential decay resembles the time profile of an idealized type III radio burst. The exponential decay can be written as $\exp \left(-t v_{120}\right)$, where $v_{120}$ is the effective collision rate due to scattering of $120 \mathrm{kHz}$ fundamental. From Fig. 3, we can estimate that $1 / v_{120} \sim 2000 \mathrm{sec}$. In the second column of Fig. 3, we present the histogram of the arrival times of the harmonic rays gathered in the longitude range from 0 to $90^{\circ}$. These time profiles show that the durations of the unscattered and scattered emissions are $\sim 350$ and $\sim 3000$ seconds, respectively. The time profile of the scattered harmonic emission also appears like that of an idealized type III burst time profile. In this case the law for the exponential decay can be written as $\exp \left(-2 t v_{60}\right)$, where $v_{60}$ is the effective collision rate due to scattering of 120 

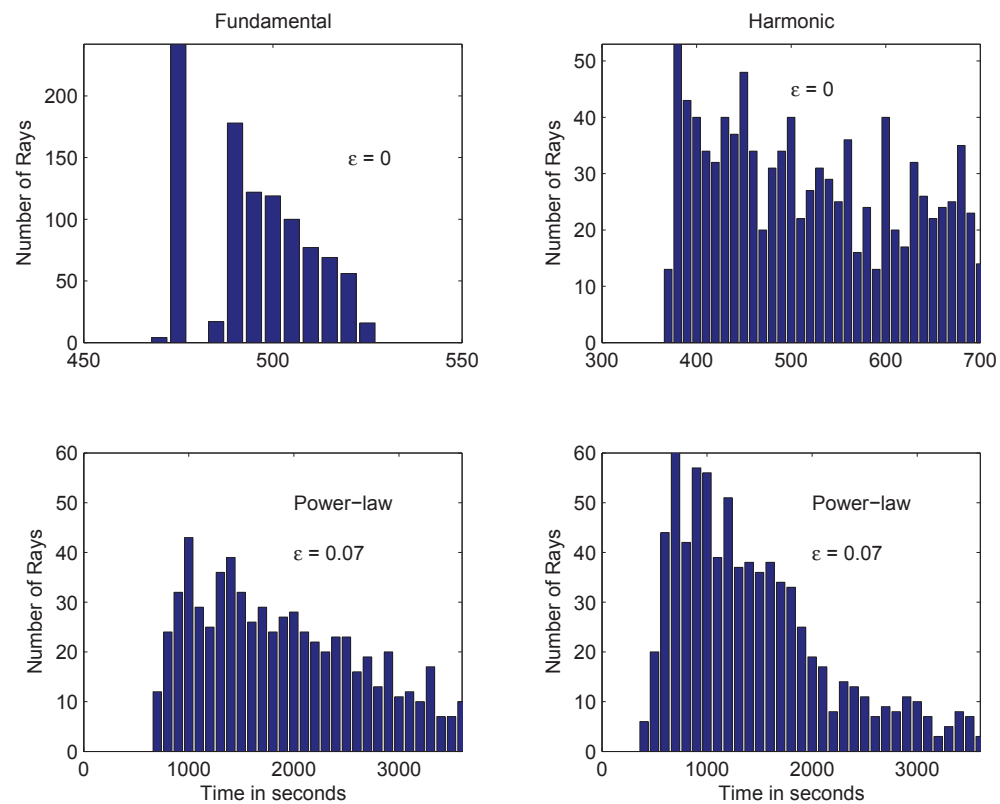

Fig. 3. The computed time profiles of the fundamental (left column) and harmonic emissions (right column) at $120 \mathrm{kHz}$ for various cases. The $\epsilon$ is equal to the level of relative density fluctuations $\frac{\Delta N_{e}}{N_{e}}$

$\mathrm{kHz}$ harmonic excited at $60 \mathrm{kHz}$ critical layer. Again, from Fig. 3, we can estimate that and $1 / v_{60}$ is $\sim 1500 \mathrm{sec}$. The computed durations of $\sim 3000 \mathrm{~s}$ for both fundamental as well as harmonic emissions are comparable to the observed durations of type III bursts at these low frequencies (Steinberg et al, 1984).

The computed time profiles also show that the fundamental $(\mathrm{F})$ and harmonic $(\mathrm{H})$ emissions arrive at the spacecraft at different times, i.e., at a given frequency a time profile may contain two peaks corresponding to $\mathrm{F}$ and $\mathrm{H}$ emissions. However, the excitation of the harmonic emission is delayed with respect to the fundamental. This delay $\Delta t$ is equal to the time taken by the beam to travel from the plasma level $\left(\sim f_{p e}\right)$ to $\frac{f_{p e}}{2}$ level

$$
\Delta t \simeq \frac{r\left(f_{p e}\right)-r\left(f_{p e} / 2\right)}{v_{b}}
$$

where $v_{b}$ is the beam speed. We use equations (1), and (6), and write the heliocentric distance as a function of the electron plasma frequency as

$$
r\left(f_{p e}\right)=19.2 f_{p e}^{-0.952} \text { AU. }
$$

This can be used to calculate $\Delta t$ for a given value of $v_{b}$. For example, a beam traveling at speeds of $v_{b}=\alpha c$ takes $\frac{89.9}{\alpha}$ seconds to travel from the fundamental layer at $0.2097 \mathrm{AU}$ (corresponding to $f_{p e}=115 \mathrm{kHz}$ ) to the harmonic layer at $0.3895 \mathrm{AU}$ (corresponding to $f_{p e}=$ $60 \mathrm{kHz}$ ). Thus, for $\alpha$ equals to 0.1 and 0.5 , the time delay is $\sim 15$ and $\sim 3$ minutes, respectively. These values are consistent with the observed time delays of the fundamental-harmonic pairs 
at high frequencies (Caroubalos \& Steinberg , 1974), as well as with those of interplanetary type III radio bursts. For typical beam velocities, the harmonic peak always occurs later than the fundamental. However, due to superposition only a single peak may appear in the time profile. Such a peak may correspond to the fundamental at the low longitudes, and to the harmonic at high longitudes. The occurrence of the fundamental emission followed by the harmonic is consistent with observations (Dulk et al ,1984; Kellogg , 1980), which implies that the time profile of a type III burst may contain a single emission peak, consisting of a mixture of the fundamental (rise part) and harmonic (peak and decay part) components. Thus, the computed time profiles and time delays between the fundamental and harmonics explain the observations that the type III bursts in the solar wind often have both fundamental and harmonic components, that in some bursts and some frequencies, the fundamental component is dominant, in others only the harmonic component is present, and in many there are two components but the components overlap considerably (Dulk et al ,1984; Kellogg , 1980; Reiner \& Stone , 1988; 1989; Reiner et al ,1992; Thejappa et al , 1993).

\subsection{Source size and displacement}

We project the exit points $(\vec{R})$ of the rays in the angular range $\beta$ and $\beta+d \beta$ onto a plane passing through the source $S$ and perpendicular to the exit direction, $\vec{T}$. The distributions of the projected points determine the sizes and displacements of the apparent sources. The equation of the plane through the source with radius vector $R_{S}$ and normal to $\vec{T}$ can be written as

$$
T_{1} x+T_{2} y+T_{3} z=D,
$$

where $D=T_{1} x_{1}+T_{2} y_{1}+T_{3} z_{1}$, and $\left(x_{1}, y_{1}, z_{1}\right)$ and $\left(T_{1}, T_{2}, T_{3}\right)$ are the components of the vectors $\vec{R}_{S}$ and $\vec{T}$, respectively. The projection of the exit point $\left(x_{2}, y_{2}, z_{2}\right)$ on this plane can be obtained from equation

$$
\frac{x-x_{2}}{T_{1}}=\frac{y-y_{2}}{T_{2}}=\frac{z-z_{2}}{T_{3}}=p ，
$$

as $\left(p T_{1}+x_{2}, p T_{2}+y_{2}, p T_{3}+z_{2}\right)$, where $p$ is a parameter. By substituting these coordinates in the equation of the plane (50), we obtain

$$
\begin{array}{r}
\vec{T} \cdot(p \vec{T}+\vec{R})=D=\vec{T} \cdot \overrightarrow{R_{s}} \\
p=\frac{\vec{T} \cdot\left(\overrightarrow{R_{s}}-\vec{R}\right)}{\vec{T} \cdot \vec{T}} .
\end{array}
$$

In the first and second columns of Fig. 4, we present the distributions of projected points of the fundamental and harmonic emissions scattered into the longitude range of 0 to $30^{\circ}$. These distributions represent the sizes of the apparent sources. The altitudes of these apparent sources can be computed as the heliocentric distances of the centroids of these distributions. For example, the $120 \mathrm{kHz}$ fundamental source located at $0.2097 \mathrm{AU}$ (corresponding to $f_{p e}=115 \mathrm{kHz}$ ) is displaced inward to the radial distance of $0.2033 \mathrm{AU}$ (the critical layer corresponding to $\sim 120 \mathrm{kHz}$ ) in the absence of density fluctuations, i.e., when $\epsilon=0$. On the other hand, the fundamental source is displaced radially outward to a distance of 0.5950 AU (critical layer corresponding to $38.8 \mathrm{kHz}$ ) due to scattering by density fluctuations with $\epsilon=0.07$. Thus, the apparent source of the fundamental lies at a radial distance corresponding to $\sim \frac{f}{3}$ layer. This altitude of the centroid of the apparent fundamental source agrees very well with the observed heights of $f / 2$ and $f / 5$ layers for type III radio bursts (Steinberg et al , 1985). In the harmonic case, the computed centroid of the apparent source shows that it is 
Fundamental source $L=0-30 \mathrm{deg}$

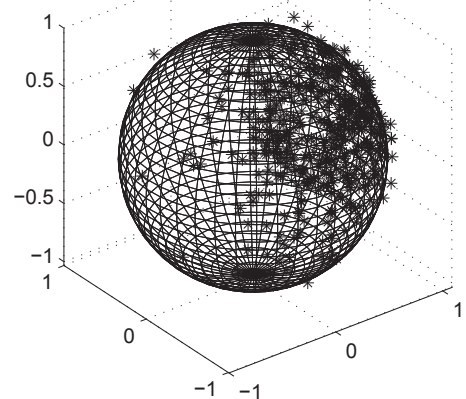

Harmonic source $L=0-30$ deg

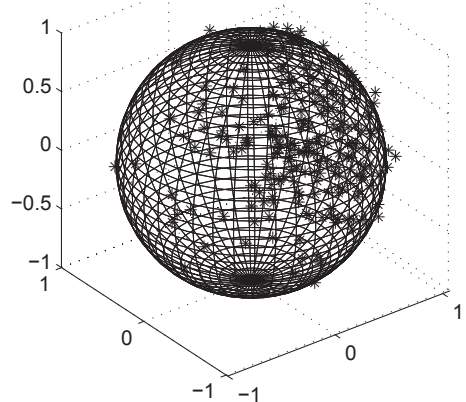

Fig. 4. The projected images of the scattered fundamental and harmonic sources.

\begin{tabular}{|c|c|c|c|c|c|c|c|}
\hline & & \multicolumn{3}{|c|}{ Fundamental } & \multicolumn{3}{|c|}{ Harmonic } \\
\hline Angles & $\epsilon=\frac{\Delta N_{e}}{N_{e}}$ & $S_{\|}$ & $S_{\perp}$ & Location & $S_{\|}$ & $S_{\perp}$ & Location \\
\hline & $\epsilon=0$ & 0.2429 & 1.1233 & 0.2033 & 6.5 & 1.96 & 0.3152 \\
\hline $0-30$ & $\epsilon=0.07$ (Power-law) & 24 & 27 & 0.5950 & 36 & 38 & 0.3993 \\
\hline & 0 & - & - & - & 6.22 & 2.13 & 0.3211 \\
\hline $0-60$ & power-law & - & - & - & 36 & 38 & 0.3993 \\
\hline
\end{tabular}

Table 1. The radial distances (location) in units of AU, the radial $S_{\|}$and transverse $S_{\perp}$ sizes (in degrees) of the refracted and scattered fundamental and harmonic sources at $120 \mathrm{kHz}$. The first column shows the range of longitudes used for these estimates

displaced inwards from its initial location of $0.3895 \mathrm{AU}$ corresponding to $60 \mathrm{kHz}$ plasma level to 0.3152- $0.3329 \mathrm{AU}$ in the absence of density fluctuations $(\epsilon=0)$. These radial distances correspond to $f_{p e} \sim 0.6 f$. When the scattering is included in the computations with $\epsilon=0.07$, the height of the apparent harmonic source is displaced to a radial distance of $0.3993 \mathrm{AU}$, corresponding to the $\sim \frac{f}{2}$ critical level. The sizes of the apparent sources are computed as the half-power widths of these distributions. When only refraction is considered with $\epsilon=0$, the size of the apparent fundamental source is $0.2429^{\circ}$, and $1.1233^{\circ}$ parallel and perpendicular to the radial direction. On the other hand, when the scattering is included with $\epsilon=0.07$, the size of the apparent source is increased to $24^{\circ}$ and $27^{\circ}$ parallel and perpendicular to the radial direction, respectively. To estimate the sizes of the harmonic sources, we have considered two ranges of longitudes, namely, 0 to $30^{\circ}$ and $30^{\circ}$ to $60^{\circ}$. When $\epsilon=0$, the size of the apparent harmonic source is $\sim 6.5^{\circ}$, and $\sim 2^{\circ}$ along and across the radial directions in both longitude ranges. When the scattering is included, the sizes in both cases are increased to $36^{\circ}$ and $38^{\circ}$ along and across the radial direction, respectively. In Table 1, we present the computed sizes and the heliocentric distances of the apparent sources. These computed sizes and heights agree very well with the observations of Steinberg et al (1984), who after analyzing a large data set reported that (1) the angular sizes of type III sources vary from $\sim 5^{\circ}$ at $1000 \mathrm{kHz}$ to $\sim 50^{\circ}$ at $100 \mathrm{kHz}$, and $\sim 60$ percent of all $100 \mathrm{kHz}$ angular sizes were between $40^{\circ}$ and $60^{\circ}$, and (2) the heliocentric distances of type III source centroids at a given frequency $f$ range from the distance where $f_{p e}=f / 2$ to that where $f_{p e}=f / 5$. 


\section{Comparison with observations}

In Fig. 5, we present an example of a multi spacecraft detection of a type II and a couple of type III radio bursts by the Unified Radio and Plasma Wave (URAP) experiment on Ulysses (Stone et al , 1992) and the Waves investigation on Wind (Bougeret et al , 1995). Ulysses is in a highly elliptical orbit out of the ecliptic plane with aphelion (perihelion) at $\sim 5.4 \mathrm{AU}(\sim 1.3 \mathrm{AU})$, the trajectory of the Wind takes it from near Earth orbits to the Lagrange point (L1), about $230 R_{E}$ upstream of Earth. The Ulysses data presented in the top panel show an intense type III burst after 12:00 on 1997/11/6 and several other weaker type III bursts. The data early on 1997/11/6 are corrupted by a poor telemetry link. The type II emission is the weaker activity (see color bar scale) starting at 18:00 and continuing to 12:00 on the next day while drifting from 200 to $100 \mathrm{kHz}$. The flare site related to these events was at S18 and W63 according to the Solar Geophysical Data. The bottom panel shows similar data from the Wind spacecraft, where the same type II and type III bursts are seen; they are detected slightly earlier because Wind is closer to the Sun (at 1 AU near the Earth) than Ulysses (at 5.3 AU). The additional emission features in the Wind data are Auroral kilometric Emission (AKR -a terrestrial radio emission appearing as short duration, broad-band feature throughout the plot) and the electron thermal noise (the horizontal feature seen in the bottom half of the panel). In the middle panel, single frequency data from the two spacecraft at $\sim 120 \mathrm{kHz}$ is plotted. The signal levels are not the same at the two spacecraft because (1) the radio bursts are directive, and (2) the distances of the sources are different for different spacecraft. However, the time profiles of type III as well as type II bursts observed at Ulysses are very similar to those observed at WIND. Note that the Ulysses data plotted in the middle panel have been shifted in time by about 35 minutes to correct for the longer propagation distance to Ulysses. (ULYSSES: Heliographic latitude $=2^{\circ}$, Heliographic longitude $=53.9^{\circ}$, Range to Sun $=5.3 \mathrm{AU}$; EARTH/WIND: Heliographic latitude $=3.8^{\circ}$, Heliographic longitude $=301.2^{\circ}$, Range to Sun $=1.0 \mathrm{AU}$ ).

We compute the distributions of rays emitted by the fundamental and harmonic sources located at (S18, W63) at altitudes of 0.2050 and 0.3895 AU and examine whether they are visible to Ulysses and Wind spacecraft, We trace the rays corresponding to both $\mathrm{F}$ and $\mathrm{H}$ components until they reach the distances comparable to those of Ulysses for (1) $\epsilon=0$ as well as (2) $\epsilon=0.07$. In Fig. 6, we show the typical distributions of these traced rays, where we also show the locations of the Ulysses and Wind spacecraft. It is clear from these distributions of traced rays that (1) when $\epsilon=0$ the fundamental is highly beamed and visible only to Ulysses spacecraft, (2) when $\epsilon=0.07$ the scattering causes the fundamental to be visible to Ulysses as well as Wind spacecraft by destroying the limiting cone, and (3) the harmonic emission is visible to both Ulysses and Wind spacecraft for $\epsilon=0$ as well as for $\epsilon=0.07$. Thus, except for the refracted fundamental, the rest of the emissions, namely scattered fundamental, unscattered harmonic as well as scattered harmonic can be visible to both Ulysses and Wind spacecraft. This indicates that the visibility of radio bursts critically depends on the coordinates of their sources.

\section{Quiet sun component}

The brightness temperature of the thermal emission from the quiet Sun is computed as

$$
T_{b}=T_{e}\left(1-e^{-\tau}\right),
$$

where

$$
\tau=\int_{s_{1}}^{s_{2}} \xi d s
$$




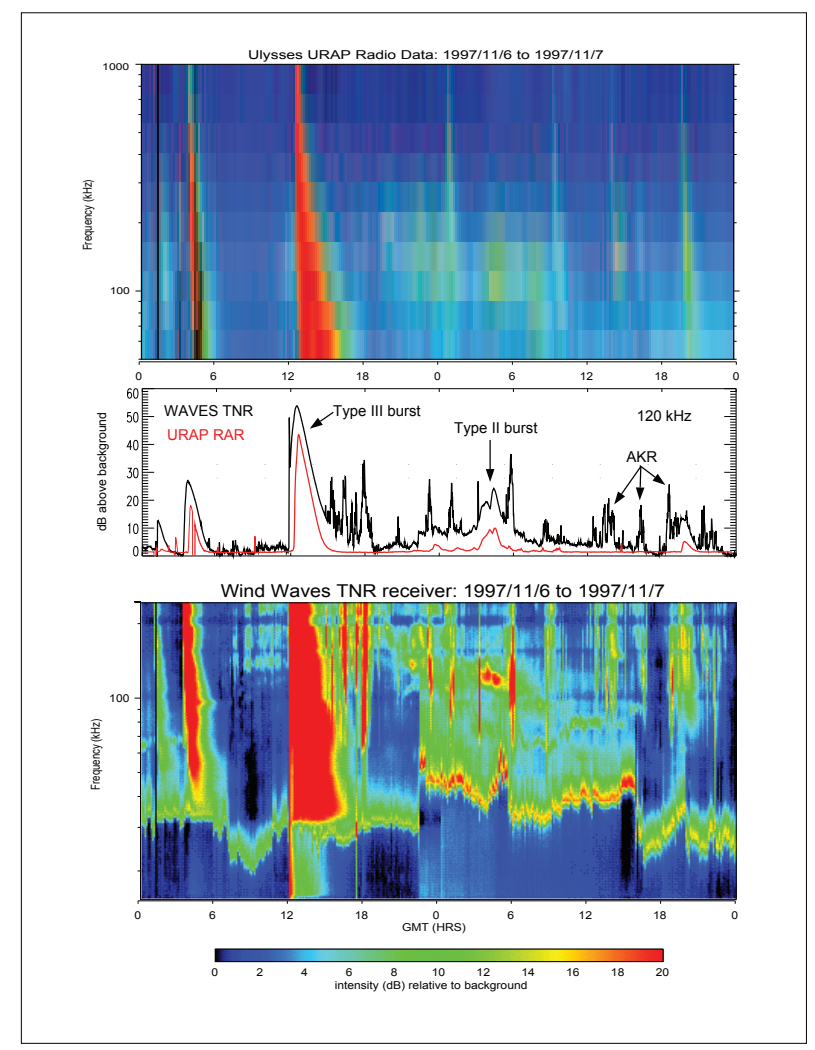

Fig. 5. Top: In the top panel, Ulysses URAP data show an intense type III burst after 12:00 on $1997 / 11 / 6$, as well as several other weaker type IIIs. The type II emission is the weaker emission band (see color bar scale) from 18:00 to 12:00 on the next day and drifting from 200 to $100 \mathrm{kHz}$. The bottom panel shows similar data from the Wind Waves instrument. In the middle panel, single frequency data from the two spacecraft at approximately $120 \mathrm{kHz}$ is plotted. Because of differing distances from the sources to the spacecraft as well as the effects of directivity, the signal levels seen for a given emission is different at the two spacecraft. Bottom: The two spacecraft are separated by more than $100^{\circ}$ in heliographic longitude, providing an ideal angular separation for studying these events.

is the optical depth, and $s_{1}$ and $s_{2}$ are the heliocentric distances of the source and the observer, respectively. The absorption coefficient per centimeter of path length, $\xi$, is defined as

$$
\xi=\frac{f_{p e}^{2} v}{f^{2} \mu c} .
$$

The brightness temperature at some point on the solar disk is determined by using equation (54), where the optical depth $\tau$ is calculated by tracing the rays (initially launched toward that point). The rays are traced from a distance of $2.5 R_{\odot}$ toward the Sun until the optical depth reaches a large value of $\sim 10$, or the ray is traveled at least $5 R_{\odot}$. The rays are launched only in the equatorial plane, where the $\mathrm{x}$-axis is directed toward the observer. Here, the positive 

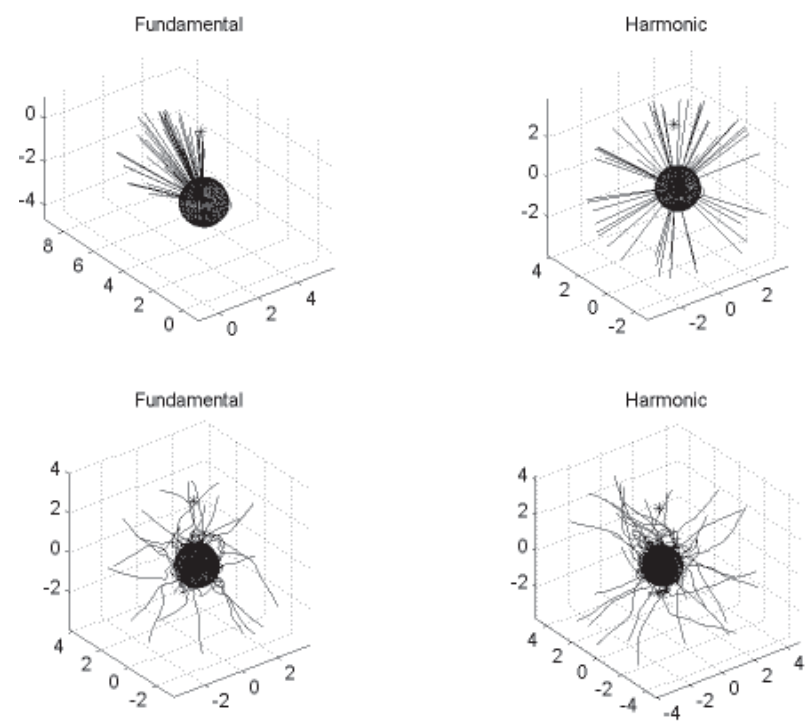

Fig. 6. The typical distributions of the refracted and scattered rays from the actual source location until they reach distances of Ulysses spacecraft. The left and right columns correspond to the fundamental and harmonic emissions, respectively. In these distribution diagrams, the locations of Ulysses and Wind are also shown as * and o, respectively.

y direction represents the west longitude. By summing the optical depths computed at each step, the total optical depth $\tau$ along each ray is calculated.

\subsection{Ray trajectories}

In Fig. 7, we present the typical trajectories of the traced rays. The top and bottom panels correspond to 34.5 and $73.8 \mathrm{MHz}$, respectively. The red trajectories correspond to the case, where only the refraction is considered. The refractive bending is very clear from these ray trajectories. The blue trajectories, on the other hand, correspond to the case, where refraction as well as scattering are considered. The random deflections of the rays are due to scattering by random density fluctuations. It is interesting to note that the scattered rays in the top panel (34.5 MHz) turn back before reaching the critical layer, i.e., much earlier than the refracted rays. This indicates that the scattering raises the East-West diameter of the Sun at $34.5 \mathrm{MHz}$. On the other hand, the turning points of the scattered rays in the bottom panel $(73.8 \mathrm{MHz})$ almost coincide with the critical layer, similar to the refracted rays. This indicates that the scattering may not affect the East-West diameters of the radio sun at $73.8 \mathrm{MHz}$.

\subsection{Brightness temperature distribution}

To calculate the brightness temperatures for different longitudes, corresponding to different values of $y$, we trace fifty rays at intervals of 0.25 solar radii for each y. For each ray, we determine the brightness temperature using the computed total optical depth of the ray. Then, we average the brightness temperatures of all rays traced in that direction. By the principle of reciprocity, this value represents the brightness temperature of thermal emission from that point on the disk. The error bars are estimated by using the variance of the measures contributing to the mean. In Fig. 8, we present the distributions of the brightness temperature 

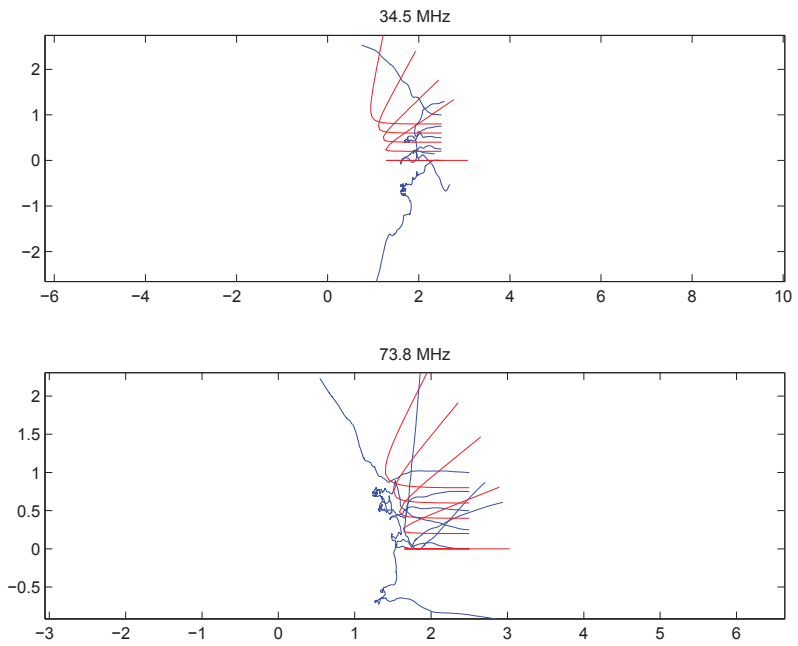

Fig. 7. Typical ray trajectories traced in the refracting (red) and refracting and scattering (blue) at 34.5 and $73.8 \mathrm{MHz}$ frequencies in a non-spherical symmetric corona. The rays are initially directed toward points on the solar disk in the intervals of $0.25 R_{\odot}$. The refractive bending is clearly seen from these trajectories. All the rays were launched along the Earth-Sun line. The random deflections of the scattered central rays are clearly visible from this figure.

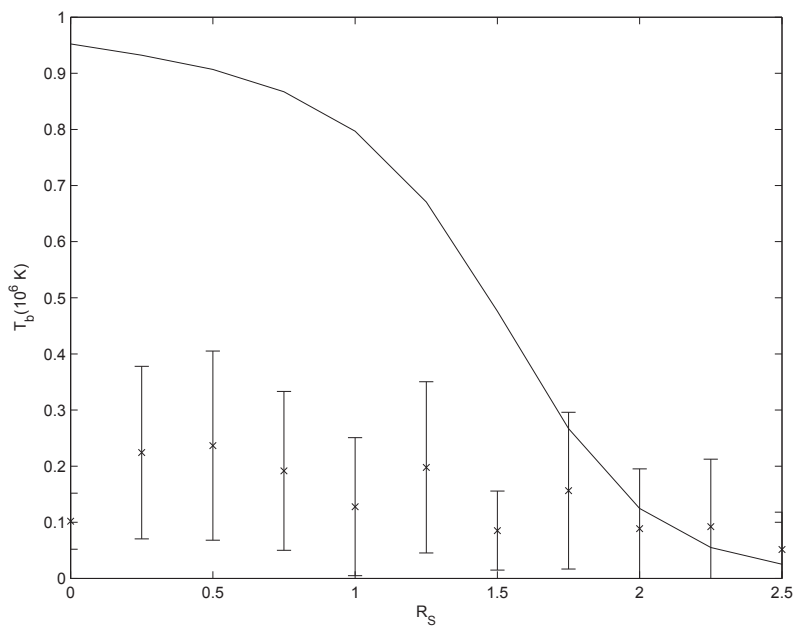

Fig. 8. Brightness temperature $\left(T_{B}\right)$ distributions for $50.0 \mathrm{MHz}$ radiation. The error bars correspond to the r.m.s deviation from the mean of the computed $T_{B}$ for individual rays. 


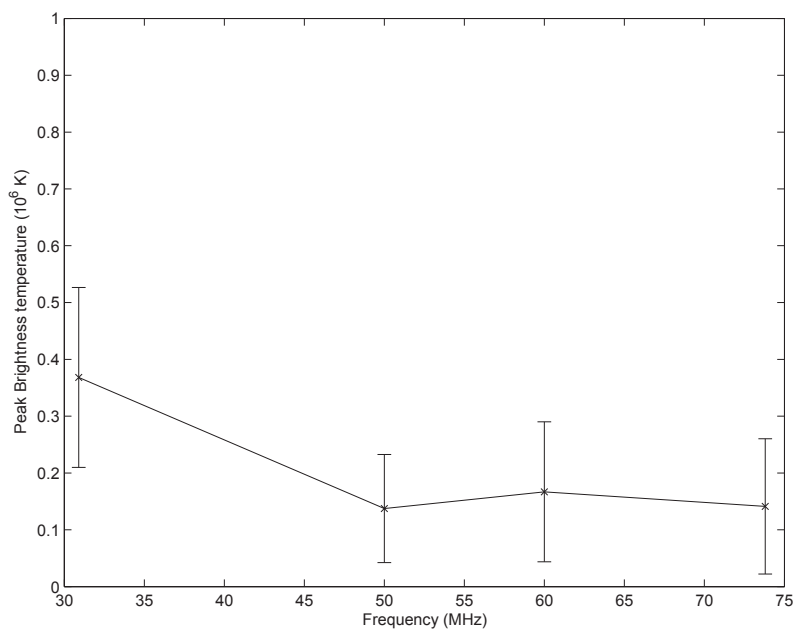

Fig. 9. Peak brightness temperature $\left(T_{B}\right)$ of the quiet Sun as a function of frequency $f$

at $50 \mathrm{MHz}$, where the solid curve corresponds to the case, where only the refraction is considered, and the points with error bars correspond to the case, where both the refraction and scattering are included in the calculations. It is interesting to note that this brightness temperature distribution (Fig. 8) resembles very much to those computed by previous authors (Aubier et al , 1971; Riddle , 1974a; Thejappa \& Kundu , 1992). We have calculated the half-power angular diameter (angular width at half-maximum) in units of arc minutes using the brightness distribution of Fig. 8, using a cubic polynomial interpolation technique. The $\mathrm{E}-\mathrm{W}$ diameter of the radio sun is $\sim 48^{\prime}$ at $50 \mathrm{MHz}$ when only the refraction is included. It is increased to $\sim 56^{\prime}$ when the scattering is added in the calculations. This value compares reasonably well with observed value of $50^{\prime}$ within the limits of error bars. As far as the brightness temperature is concerned, the peak value when scattering was absent was $9 \times 10^{5}$ $\mathrm{K}$, which is reduced to $\sim 2.3 \times 10^{5} \mathrm{~K}$ when the scattering was included, i.e., the brightness temperature is reduced almost by $50 \%$ due to scattering. We have statistically computed the most probable central brightness temperatures (zero longitude, or $y=0$ ) for four different frequencies $(30.9,50,60,73.8 \mathrm{MHz})$, which are presented as the computed spectrum in fig. 9 . The shape of the spectrum of thermal emission is preserved in the considered frequency range, even in the presence of density fluctuations by remaining almost steady at these frequencies in the limits of error bars. The central brightness temperatures are $3.5 \times 10^{5} \mathrm{~K}, 1.4 \times 10^{5} \mathrm{~K}$, $1.7 \times 10^{5} \mathrm{~K}$, and $1.4 \times 10^{5} \mathrm{~K}$ at $30.9,50,60$ and $73.8 \mathrm{MHz}$, respectively.

\section{Discussion}

We have described Monte Carlo methods used in solar radio astronomy and demonstrated how to use them to explain some of the unusual characteristics of solar radio bursts as well as quiet sun radio emissions. We have examined the effects of propagation on the directivities, time profiles, sizes and positions of the $120 \mathrm{kHz}$ radio burst emissions located at $\sim 115 \mathrm{kHz}$ and $60 \mathrm{kHz}$ plasma levels. The results are applicable for any fundamental (F) and harmonic $(\mathrm{H})$ emissions. We have also examined to what extent the scattering is responsible for the 
very low brightness temperatures and very large apparent source sizes of the quite sun radio emissions.

\subsection{Type III radio bursts}

First, we have examined whether the widespread visibility of the fundamental and harmonic components is due to scattering. For such purpose, we have assumed that the type III bursts are emitted by isotropic point sources by ignoring the effects of finite source sizes and different shapes of the emission patterns. Even for such simplistic input parameters, the simulations have shown that the scattering increases the visibilities of the fundamental and harmonic emissions from $18^{\circ}$ to $100^{\circ}$, and from $80^{\circ}$ to $150^{\circ}$, respectively. The assumption of the isotropic emission patterns does affect the results because, first of all the refractive focusing does not depend on the shape of the emission patterns, and second of all the emission patterns are completely destroyed by the scattering. On the other hand, the introduction of a finite source size may increase the visibility as well as the sizes of the apparent sources.

The second question, we have examined is the effect of scattering on the time profiles of radio bursts. We have constructed the time profiles of the isotropic point sources using the arrival times of the scattered rays at $1 \mathrm{AU}$, and found them to be very similar to the observed type III burst profiles, i.e., a large fraction of the durations and exponential decays is due to propagation effects, especially due to scattering. Thus, these simulations conclusively show that we can use the durations and decay constants obtained from the observed time profiles to derive the characteristics of the electron beam and the electron temperature of the ambient plasma, only if we correct them for the propagation effects.

The third question concerns the connection between the mode of the observed emission and the propagation effects. We have shown that when the level of density fluctuations is low, the fundamental emission is dominant in a narrow range of angles around the radial direction, i.e., along the direction of the beam. Thus the identification of the mode of the observed emission as the fundamental, or a mixture of a strong fundamental and a weak harmonic by an independent technique can serve as a good indicator for the oncoming flare electrons or CME driven shock accelerated electrons at the spacecraft. On the other hand the scattering allows both the fundamental and harmonic modes to be equally visible at low longitudes, and only harmonic to be visible at high longitudes. We have also examined the question of time delay between the fundamental and harmonic emission peaks in the observed time profiles, and have shown that it depends critically on the speed of the electron beam and the position of the observer. The computations have also indicated that the usually observed single emission peak consisting of a mixture of the fundamental and harmonic emissions in a given time profile can be easily accounted for by the time delays due to propagation effects, location of the observer, and the beam speed.

The fourth question is concerned with the effects of refraction and scattering on the sizes and positions of the fundamental and harmonic emissions. We have shown that the propagation effects increase the sizes and heights of the radio sources considerably. The refraction lowers the heights of the centroids of the fundamental as well as harmonic sources, contradicting the observations that the heliocentric distances of the $100 \mathrm{kHz}$ type III burst source centroids at a given frequency f range from the distance where $f_{p e}=f / 2$ to that where $f_{p e}=f / 5$. This suggests that the refraction alone can not account for either the observed source sizes or source heights. When the scattering is included, the angular sizes are increased to $\sim 25^{\circ}$ for the fundamental and $\sim 37^{\circ}$ for the harmonic, and the heliocentric distances of their centroids increased to $f_{p e}=f / 3$ and $f_{p e} \sim f / 2$ levels, respectively. These values agree very well with 
observations. One should note that higher value of $\epsilon$, for example, 0.1 , may provide a still better agreement with observations, yielding larger source sizes and higher altitudes.

The distributions of the traced rays from the actual location of the source to distances of Ulysses show that the scattered fundamental, refracted harmonic as well as the scattered harmonic can easily account for the widespread visibility of radio bursts to Ulysses and Wind spacecraft. This also shows that the location of the source will have considerable influence on its visibility.

\subsection{Quiet sun radio emission}

The radio emission at meter and decameter wavelengths from the solar corona during the quiet periods of solar activity is one of the best examples of the thermal continuum emissions in nature. By measuring the brightness temperatures of this emission at different wavelengths, in principle, we can obtain the electron temperature in the corona at different heights. However, the observations have shown that the quiet sun radio emission exhibits very peculiar behavior. The East-West diameters are larger than the expected values from the thermal emission. Because of this peculiar behavior, the radio methods do not yield the correct electron temperatures. Earlier investigations (Aubier et al , 1971; Thejappa \& Kundu , 1992; 1994) have shown that the scattering probably is responsible for such unusual behavior. However, in those studies, spherical symmetric models for the electron density and Gaussian power spectrum for the the density fluctuations were used. Those models do not represent the real solar conditions.

In this study, we have used the non-spherical symmetric density model for the quiet corona, which shows a bulge in equatorial region and a sort of compression in polar regions. This density distribution represents the actual observed shape of the quiet corona. Extensive observations have also shown that the spectrum of density fluctuations are correctly described by a power-law. The flat part of this spectrum with spectral index, $\alpha=3$ contains most of the power. In this spectral range, the geometric optics approximation is also valid. We have used a range of density scales, ranging from 50 to $75 \mathrm{~km}$ with axial ratio of 10 . These are very close to the scale of $5 \times 10^{-5} R_{\odot}$ used by previous authors. We have derived an expression for the angular deflection suffered by a ray due to power-law spectrum with $\alpha=3$ and with spatial scales of $\frac{L_{i}}{L_{o}} \sim 0.7$ and have shown that it is almost identical to that of Gaussian spectrum. The observations also indicate that the relative level of density fluctuations, $\epsilon=\frac{\Delta N_{e}}{N_{e}}$ is 0.1 . We have used this value in the simulations. We have shown that these values cause a reduction in the brightness temperatures by almost by an order of magnitude by increasing the sizes considerably. These two results are consistent with observations. We have used for example, a value of $T_{e}=10^{6}$ for all the calculations. However, in order to extract an accurate information about the electron temperature $T_{e}$, from the observed brightness temperatures, one should use the models for both electron density as well as electron density fluctuations based on observations (preferably real-time), and statistically calculate the brightness temperatures for various electron temperatures at different wavelengths. The value of $T_{e}$ which yields a correct value of $T_{B}$ will represent the correct electron temperature at a given height. Sometimes, abnormally low brightness temperatures are observed as reported by Thejappa \& Kundu (1992). These cases may be due to equatorial coronal holes with very low electron temperatures. For example, using the data from two SOHO spectrometers CDS and SUMER, David et al (1998) have shown that in a polar coronal hole, the electron temperatures are around $0.8 \mathrm{Mk}$ close to the limb, rising to a maximum of less than $1 \mathrm{MK}$ at $1.15 R_{\odot}$, then 
falling around $0.4 \mathrm{MK}$ at $1.3 R_{\odot}$ and $0.3 \mathrm{MK}$ at $1.6 R_{\odot}$ (Wilhelm et al , 1998). In these cases, the scattering definitely can lead to very low brightness temperatures.

\section{Conclusions}

The main results from these Monte Carlo simulations are: (1) the widespread visibility of radio bursts is due to scattering of radio waves by density fluctuations, (2) the scattered fundamental and harmonic emissions produce time profiles which look very much like the idealized type III radio bursts indicating that the duration of the beam and collisional decay constants can be derived from the observed time profiles only after correcting for the propagation effects, (3) the identification of the emission modes in the type III burst time profile, namely the fundamental in the rise part followed by the harmonic in the decay part of the time profile can be accounted for by the scattering, (4) the sizes and heights of the apparent sources derived using the distributions of scattered rays from the isotropic point sources agree very well with observed values, (5) the scattering at meter-decameter wavelengths leads to a considerable reduction in the central brightness temperatures, (6) although scattering causes the reduction in the central brightness temperatures, the resultant spectrum, i.e., the peak brightness temperature as a function of the frequency remains very similar to the thermal spectrum of electromagnetic radiation, and (7) by knowing the density distribution, and the parameters of density fluctuations during the radio observations, we can determine the electron temperatures of the solar corona using the Monte Carlo simulations.

The Monte Carlo simulation methods developed in this study are very general. These techniques can be used to study the propagation of waves in any refracting and scattering medium. The diagnostics developed in this study to calculate the directivities, time profiles, sizes and positions of the radio sources can be used in a variety of contexts in solar radio astronomy. Since the scattering is probably responsible for the reduction in the intensities of thermal emission from the quiet Sun, and for the increase in the source size of the quiet Sun, a proper simulation of this process can yield an accurate determination of the electron temperature, $T_{e}$.

\section{Acknowledgements}

The research of Thejappa Golla is supported by the NASA grants NNX08AO02G and NNX09AB19G.

\section{References}

Armstrong, J. W., Coles, W.A., Kojima, M., \& Rickett, B. J. (1990), Astrophys. J, 358, 685.

Aubier, M., Leblanc, Y., \& Boischot, A. (1971), Astron. Astrophys., 12, 435.

Bale, S. D., Reiner, M. J., Bougeret, J.-L., Kaiser, M. L., Krucker, S., Larson, D. E., \& Lin, R. P. (1999), Geophys. Res. Lett., 26, 1573.

Bastian, T. S., (1994), Astrophys. J., 426, 774.

Bavassano, B., \& Bruno, R. (1995), J. Geophys. Res, 100, 9475.

Bougeret, J. L., Fainberg, J., \& Stone, R. G. (1984a), Astro. Astrophys., 141, 17.

Bougeret, J.-L., King, J. H., \& Schwenn, R. (1984b), Sol. Phys., 90, 401.

Bougeret, J.-L. et al., (1995), Spa. Sci. Rev., 71, 231.

Bracewell, R. N., \& Preston, G. W. (1956), Astrophys. J., 123, 14.

Cairns, I. H. (1998), Astrophys. J., 506, 456.

Caroubalos, C., Aubier, M., Leblanc, Y \& Steinberg, J. L. (1972), Astron. Astrophys., 16, 374. 
Caroubalos ,C., \& Steinberg, J. L. (1974), Astron. Astrophys., 32, 245.

Caroubalos, C., Poquerusse, M., \& Steinberg, J. L. (1974), Astron. Astrophys., 32, 255.

Chandrasekhar, S. (1952), Mon. Noti. Roy. Astron. Soc., 112, 475.

Coles, W. A., \& Filice, J. P. (1985), J. Geophys. Res., 90, 5082.

Coles, W. A., Frehlich, R. G., Eickett, B. J., \& Codona, J. L. (1987), Astrophys. J., 315, 666.

Coles, W. A., \& Harmon, J. K. (1989), Astrophys. J., 337, 1023.

Coles, W. A., Liu, W., Harmon, J. K. \& Martin, C. L., (1991), J. Geophys. Res., 96, 1745.

Coles, W. A., Rao, A. P., \& Ananthakrishnana, S., (2002) Solar Wind 10, Pisa.

David, C., Gabriel, A. H., Bely-Dubau, F., Fludra, A., Lemaire, P., \& Wilhelm, K., (1998), Astron. Astrophys., 336, L90.

Dulk, G. A., Steinberg, J. L., \& Hoang, S. (1984), Astro. Astrophys., 141, 30.

Dulk, G. A., Steinberg, J.-L., Lecacheux, A., Hoang, S., \& MacDowall, R. J. (1985), Astron. Astrophys., 150, L28.

Dulk, G. A., Leblanc, Y., Bougeret, J. L., \& Hoang, S. (1996), Geophys. Res. Lett., 23, 1203.

Efimov, A. I., Chashei, I. V., Bird, M. K., Samoznaev, L. N., \& Plettemeier, D. (2005), Astro. Rep., $49,485$.

Fainberg, J.,\& Stone, R. G. (1970), Sol. Phys., 15, 222.

Fainberg, J., Evans, L. G., \& Stone, R. G. (1972), Science, 178, 743.

Fainberg, J., \& Stone, R. G. (1974), Spa. Sci. Rev., 16, 145.

Fitzenreiter, R. J., Fainberg, J., Bundy, R. B. (1976), Sol. Phys., 46, 465.

Fokker, A. D. (1965), Bul. Astro. Inst. Neth., 18, 111.

Ginzburg, V. L., \& Zheleznyakov, V. V. (1958), Sov. Astro., 2, 653.

Golap, K., \& Sastry, Ch. V., (1994), Sol. Phys., 295, 150.

Grall, R. R., Coles, W. A., Spangler, S. R., Sakurai, T., \& Harmon, J. K., (1997), J. Geophys. Res., $102,263$.

Guhathakurta, M., Holzer, T. E., \& MacQueen, R. M., (1996), Astrophys. J., 458, 817.

Gurnett, D. A., \& Anderson, R. R. (1976), Science, 194, 1159.

Gurnett, D. A., Baumback, M. M.,\& Rosenbauer, H. (1978), J. Geophys. Res., 83, 616.

Haddock, F. G.,\& Graedel, T. F. (1970), Astrophys. J., 160, 293.

Hartz, T. R. (1969), Plan. Spac. Sci., 11, 115.

Haselgrove, J. (1963), J. Atmos. Terr. Phys., 25, 397.

Hoang, S., \& Steinberg, J. L., (1977) Astro. Astrophys., 58, 287.

Hoang, S., Maksimovic, M., Bougeret, J.-L., Reiner, M. J., \& Kaiser, M. L. (1998), Geophys. Res. Lett., 25, 2497.

Hollweg, J. (1968), Astron. J, 73, 972.

Hughes, M. P., \& Harkness, R. L. (1963), Astrophys. J., 138, 239.

Jaeger, J. C., \& Westfold, K. C. (1950), Austr. J. Res., (A)., 2, 322.

Kaiser, M. L. (1975), Sol. Phys., 45, 181.

Kellogg, P. J. (1980), Astrophys. J., 236, 696.

Kundu, M. R., (1965), Solar Radio Astronomy, Interscience Publishers.

Lacombe, C., Harvey, C. C., Hoang, S., Mangeney, A., Steinberg, J.-L., \& Burgess, D. (1988), Ann.Geophys., 6, 113.

Lantos, P., (1980) in Radiophysics of the Sun (M. R. Kundu and T. Gergeley eds.), Proceedings of IAU Symposium No. 86.

Lantos, P., (1998), Solar Physics with Radio observations, proc. Nobeyama Symposium, NRO Report 479.

Leblanc, Y. (1973), Astrophys. J. Lett., 14, 41. 
Lecacheux, A., Steinberg, J.-L., Hoang, S., \& Dulk, G. A. (1989), Astron. Astrophys., 217, 237.

Lee, L. C., \& Jokipi, J. R. (1975), Astrophys. J., 196, 695.

Lin, R. P., Levedahl, W. K., Lotko, W., Gurnett, D. A., \& Scarf, F. L. (1986), Astrophys. J., 308, 954.

MacDowall, R. J. (1983), M. S. Thesis, University of Maryland.

Manoharan, P. K., Ananthakrishnan, S., \& Rao, A. P. (1988), Proc. Sixth International Solar Wind Conf. Vol. 1 (Boulder, NCAR), 55.

Narayan, R., Anantharamaiah, K. R., \& Cornwell, T. J. (1989), Mon. Not. Roy. Astron. Soc., 241, 403.

Newkirk, G. A., (1961), Astrophys. J., 133, 983.

Poquerusse, M., Steinberg, J. L.. Caroubalos, C., Dulk, G. A., \& MacQueen, R. M. (1988), Astron. Astrophys., 192, 323.

Ramesh, R., Nataraj, H. S., Kathiravan, C., \& Sastry, Ch. V. (2006), Astrophys. J., 648, 707.

Reiner, M. j., \& Stone, R. G. (1988), Astron. Astrophys., 206, 316.

Reiner, M. j., \& Stone, R. G. (1989), Astron. Astrophys., 217, 251.

Reiner, M. J., Fainberg, J., \& Stone, R. G. (1992), Astrophys. J., 394, 340.

Reiner, M. J., Fainberg, J., Kaiser, M. L., \& Stone, R. G. (1998), J. Geophys. Res., 103, 1923.

Rickett, B. J. (1977), Ann. Rev. Astron. Astrophys., 15, 479.

Riddle, A. C. (1972), Proc. Astron. Soc. Austr., 1972,2, 98.

Riddle, A. C. (1974a), Sol. Phys., 36, 375.

Riddle, A. C. (1974b), Sol. Phys., 35, 153.

Rytov, S. M., Kravtsov, Yu. A., \& Tatarskii, V. I. (1989), Principles of Statistical Radiophysics. vol. 4. Wave Propagation Through Random Media. Springer-Verlag.

Sastry, Ch. V. (1994), Sol. Phys., 150, 285.

Sawyer, C., \& Warwick, J. W. (1987), Astron. Astrophys., 206, 316.

Sharma, A., Vizia Kumar, D., \& Ghatak, A. K. (1982), Appl. Opt., 21.

Sheridan, K. V., \& McLean, D. J. (1985), (in) Solar Radiophysics (D. J. McLean and N. R. Labrum, eds), Cambride University press, Cambridge.

Smerd, S. F. (1950), Australian J. Sc., Res., A3, 34.

Smith, D. F. (1970), Sol. Phys., 15, 202.

Spangler, S. R., \& Sakurai, T. (1995), Astrophys. J., 445, 999.

Spangler, S. R., Kavars, D. W., Kortenkamp, P. S., Bondi, M., Mantovani, F., \& Alef, W. (2002), Astron. Astrophys., 384, 654.

Spangler, S. R. (2002), Astrophys. J., 576, 997.

Steinberg, J.-L., Aubier-Giraud, M., Leblanc, Y., \& Boischot, A. (1971), Astro. Astrophys., 10, 362.

Steinberg, J. L. (1972), Astro. Astrophys., 18, 382.

Steinberg, J.-L., Dulk, G. A., Hoang, S., Lecacheux, A., \& Aubier, M. G. (1984), Astro. Astrophys., $140,39$.

Steinberg, J.-L., Hoang, S., \& Dulk, G. A. (1985), Astro. Astrophys., 150, 205.

Stone, R. G., et al. 1992, Astron. Astrophys. Supp., 92, 291.

Subramanian, K. R. (2004), Astro. Astrophys., 426, 329.

Tarnstrom, G. I., \& Philip, K. W. (1972), Astro. Astrophys., 17, 267.

Thejappa, G., \& Kundu, M. R. (1992), Sol. Phys., 140, 19.

Thejappa, G., Lengyel-Frey, D., Stone, R. G., \& Goldstein, M. L. (1993), Astrophys. J., 416, 831.

Thejappa, G., \& Kundu, M. R. (1994), Sol. Phys., 149, 31.

Thejappa, G., \& MacDowall, R. J. (1998), Astrophys. J., 498, 465. 
Thejappa, G., MacDowall, R. J., \& Kaiser, M. L. (2007), Astrophys. J., 671, 894.

Thejappa, G.,\& MacDowall, R. J. (2008a), Astrophys. J., 676, 1338.

Thejappa, G.,\& MacDowall, R. J. (2008b), (in) Turbulence, Dynamos, Accretion Disks, Pulsars and Collective Plasma processes, Astrophysics and Space Science proceedings, part VI, 311-328, DOI:10.1007/978-1-4020-8826-1-21.

Thejappa, G.,\& MacDowall, R. J. (2010), Astrophys. J., 720, 1395.

Tu, C. Y., \& Marsch, E. (1994), J. Geophys. Res., 9921,481.

Wilhelm, K., Marsch, E., Dwivedi, B. N., Hassler, D. M., Lemaire, P., Gabriel, A. H., \& Huber, M. C. E. (1998), Astrophys. J., 500, 1023.

Wohlmuth, R., Plettemeier, D., Edenhofer, P., Bird, M. K., Efimov, A. I., Andreev, V. E., Samoznaev, L. N., \& Chashei, I. V. (2001), Spa. Sci. Rev., 97, 9.

Woo, R., Armstrong, J. W., Bird, M. K., \& Patzold, M. (1995), Geophys. Res. Lett., 22, 329.

Zheleznyakov, V. V., \& Zaitsev, V. V. (1970), Sov. Astron., 14, 250. 


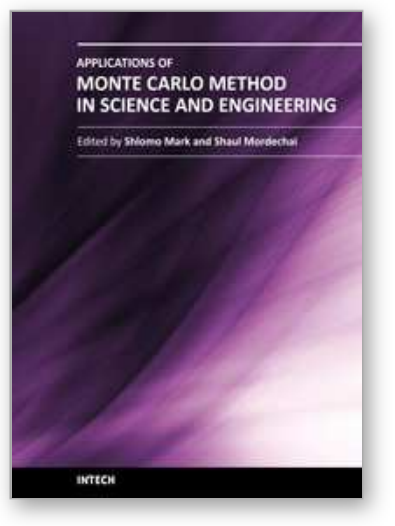

\section{Applications of Monte Carlo Method in Science and Engineering \\ Edited by Prof. Shaul Mordechai}

ISBN 978-953-307-691-1

Hard cover, 950 pages

Publisher InTech

Published online 28, February, 2011

Published in print edition February, 2011

In this book, Applications of Monte Carlo Method in Science and Engineering, we further expose the broad range of applications of Monte Carlo simulation in the fields of Quantum Physics, Statistical Physics, Reliability, Medical Physics, Polycrystalline Materials, Ising Model, Chemistry, Agriculture, Food Processing, X-ray Imaging, Electron Dynamics in Doped Semiconductors, Metallurgy, Remote Sensing and much more diverse topics. The book chapters included in this volume clearly reflect the current scientific importance of Monte Carlo techniques in various fields of research.

\section{How to reference}

In order to correctly reference this scholarly work, feel free to copy and paste the following:

G. Thejappa and R. J. MacDowall (2011). Monte Carlo Simulations in Solar Radio Astronomy, Applications of Monte Carlo Method in Science and Engineering, Prof. Shaul Mordechai (Ed.), ISBN: 978-953-307-691-1, InTech, Available from: http://www.intechopen.com/books/applications-of-monte-carlo-method-in-science-andengineering/monte-carlo-simulations-in-solar-radio-astronomy

\section{INTECH}

open science | open minds

\section{InTech Europe}

University Campus STeP Ri

Slavka Krautzeka 83/A

51000 Rijeka, Croatia

Phone: +385 (51) 770447

Fax: +385 (51) 686166

www.intechopen.com

\section{InTech China}

Unit 405, Office Block, Hotel Equatorial Shanghai

No.65, Yan An Road (West), Shanghai, 200040, China

中国上海市延安西路65号上海国际贵都大饭店办公楼 405 单元

Phone: +86-21-62489820

Fax: $+86-21-62489821$ 
(C) 2011 The Author(s). Licensee IntechOpen. This chapter is distributed under the terms of the Creative Commons Attribution-NonCommercialShareAlike-3.0 License, which permits use, distribution and reproduction for non-commercial purposes, provided the original is properly cited and derivative works building on this content are distributed under the same license. 